\title{
Various Methods for Estimation of Biomechanical Properties of Bone to Determine Strength of Bone
}

\author{
Sanvi Bhise* and Raviraj Havaldar \\ ${ }^{1}$ Department of Biomedical Engineering, KLE Dr M S Sheshgiri College of Engineering, Belagavi India.
}

\begin{abstract}
Bone is a living growing tissue on which different loads are imposed and is adaptive to changes in loading conditions. Bone mechanics in an interdisciplinary area of research where mechanical strength of bone under extreme force is studied. The present manuscript study is based upon biomechanical properties of bone for determination of bone strength. The results of the study highlight the methods for estimation of load distribution upon growing mass of calciferous structure and thus the varying degree of strength of the osteon. The advances in the biomedical engineering in the field of bone mechanics are using finite element modelling, a computer technique based on mechanism of continuum (domain) mechanism as an effective tool for modelling and simulation nowadays. To provide details regarding development in the area of application of finite element analysis for finding bone mechanics in the last decade, this paper presents state of art review on different methods to find bone mechanics. The use of different invasive and non-invasive methods helped to provide comprehensive diagnostic study for bone locomotary strengths and the physical tensile strength and structural design of different bone samples reveal the strength statistics, thus proving useful for fall risk assessment and other fracture risk analysis.
\end{abstract}

KEY WORDS: BONE MECHANICS, BIOMEDICAL IMAGING, FINITE ELEMENT MODEL (FEM), FRACTURE RISK, INVASIVE AND NON-INVASIVE TECHNIQUE.

\section{INTRODUCTION}

Bone is a living material which is capable to repair it-self and adjust to changes in loading conditions. The present study is based upon the combination of bone strength diagnosis by combination of invasive as well as noninvasive tests to correct bone problems due to mechanical shear and strain. This study is of clinical importance for mitigating hazards after a risk assessment for falls and fractures due to impaired bone locomotive functions (Kong et al., 2020), (Claes et al.,2012) With increase in age

\section{ARTICLE INFORMATION}

*Corresponding Author: samidhapusegaonkar@gmail.com Received 9th Oct 2020 Accepted after revision 30th Dec 2020 Print ISSN: 0974-6455 Online ISSN: 2321-4007 CODEN: BBRCBA

Thomson Reuters ISI Web of Science Clarivate Analytics USA and Crossref Indexed Journal

\section{Clarivate
Analytics}

NAAS Journal Score 2020 (4.31)

A Society of Science and Nature Publication,

Bhopal India 2020. All rights reserved.

Online Contents Available at: http//www.bbrc.in/

Doi: http://dx.doi.org/10.21786/bbrc/13.13/11 the bone strength decreases and the risk for hip fracture increases exponentially. Hospitalization is must for hip fractures and sometimes it requires surgery which is very expensive and cumbersome (Op et al.,2011) Hip fractures may sometime lead to disability which further result in increased mortality rate especially in old aged person.

The skeletal fragility if left undiagnosed and untreated can lead to serious health complications and in severe instances lead to mortality or permanent loss of function of locomotive functions. Thus the bone strength can be determined with proper knowledge of localized muscle mass for behavioral mechanical movement study. The studies are well aligned to bone structure and nutritional status of dietary interventions that escalate strength of the bone material in higher stress situations. Regardless of these problems there is no cost effective, fast and noninvasive technique for determination of bone strength. Mechanical characteristic of bone reflect the quality of bone (Kong et al.,2020) Mechanical properties of bone can be estimated by invasive and non - invasive techniques.

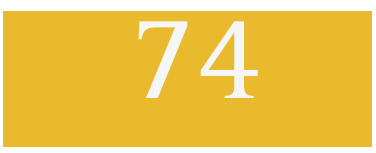


Invasive technique is testing the sample for its strength using Universal Testing Machine, this method requires actual bone sample of human to be tested. Taking the bone out of the body of a human being to test its mechanical properties is not feasible. Another method to test mechanical properties of bone is a non - invasive technique which is nothing but imaging based method for assessment of mechanical properties of bone. To describe bone mechanics shape and Bone Mineral Density (BMD) are the important parameters (Taghizadeh et al., 2016). Clinically areal bone density is obtained by DEXA (Dual energy X-ray) technique and volumetric bone density is obtained by QCT (Quantitative Computed Tomography). These imaging techniques also provide shape of a bone which is also as equally as important as BMD in determining mechanical properties of bone.

FEA divides Region of Interest (RoI) into several sub regions called as finite elements, each of these elements is then solved independently and the result is obtained by combining the results of all individual elements. More correct are the boundary conditions more accurate are the results (Parashar et al.,2016) Basic Block diagram of FEM of bone (Kong et al.,2020) is shown on (Figure 1) which shows the data profile for Finite element modeling of Bone. It is based on analysis of bone strength which involves the pre-processor analysis, the solution for rectifying the precursor effects as well as the post process stage which highlights the sprain and the mechanical load that can be beared by the bony skeletal system.

Figure 1: FEM of Bone

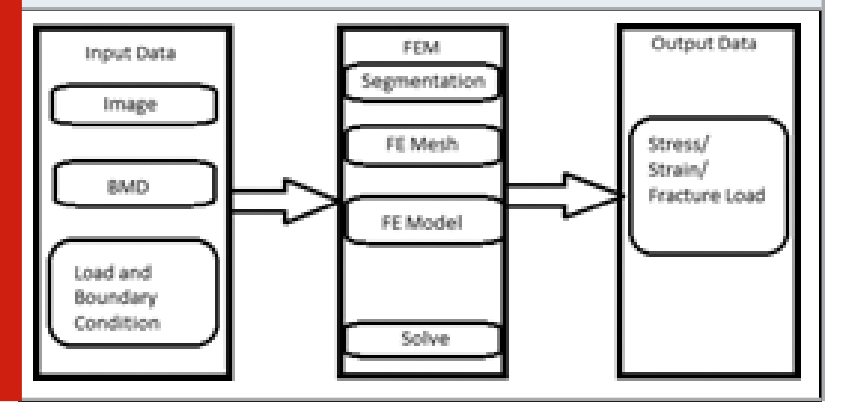

This paper provides a brief review on different methods available to determine mechanical properties of bone. There are 2 types of determination of bone mechanics - 1. Invasive 2. Non-invasive. Existing methods use combination of invasive (mechanical loading) test and non- invasive (FEM). Along with this multilevel mechanical loading test, microstructure methods and FEM reconstruction can be used to estimate bone mechanics (Yu et al.,2014) Different mechanical tests such as compression, tensile (Kong et al.,2020) bending, and torsional (Lopes et al.,2017) can be performed on bone considering it as linear elastic material; these tests are invasive in nature. QCT, DEXA are the clinical noninvasive methods to determine BMD, these techniques are costly.
Bone quality analysis using BMD and bone mechanics is an emerging research topic, so to study bone mechanics FEM can be used widely which is an effective tool to model and simulate biomaterials (Kong et al.,2020) This paper mainly focuses upon the testing of bone mechanical strength by different models of invasive as well as noninvasive methods, which helps in improving the efficacy of the methodology. Thus the use of invasive models can be done only on surgically removed bones and preserved bone structures whereas the current problems in bone movement due to declining strength can be done with non-invasive methods without any surgical interventions for peeping down into the interior body. This novel approach of combination of comprehensive tests improves the precision of the results and the present osteo problems can be diagnosed and preventive measures to avoid its progression can be done.

\section{MATERIAL AND METHODS}

The different literature works related to this topic highlight the various types of mechanical loads in terms of defining factors such as longitudinal, transverse as well as shear stress on bone tissues. The various classifications are a based upon Compression, Tension, Poisson's Ratio as well as Modulus. The measurement of these factors clearly highlights the bone strength. Bone mineral density and porosity are other important factors for strength determination.

In addition to this load tolerance as well as stress strain relationship is used for strength measurement in terms of bone material and ultimately stress tolerance till fracture can be avoided. Also some biological factors mineralization of bone tissues as well as collagen content is important as per clinical manifestations ultimately stress tolerance till fracture can be avoided. Two techniques are there to Estimate Bone mechanics which is broadly classified into 2 types - invasive and non - invasive.

Figure 2: Mechanical Tests

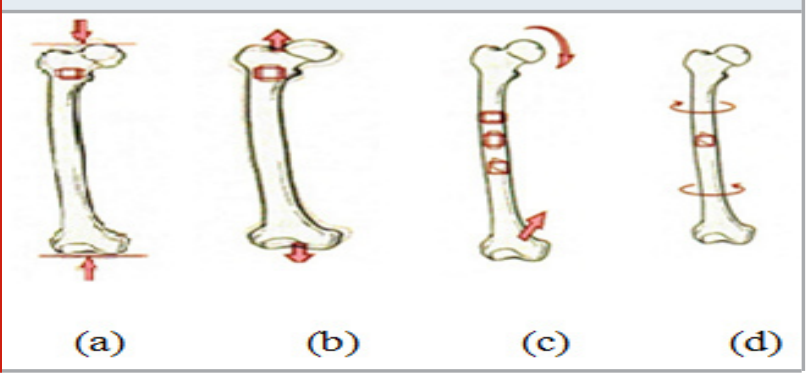

2.1 Invasive Techniques For Bone Mechanics: Invasive technique is testing the sample for its strength outside the body using Universal Testing Machine, this method requires actual bone sample of human to be tested. 
There are 4 mechanical tests which are performed on bone; they are compression test, tensile test, bending test and torsional test. As shown in (Figure 2(a) tensile test (b) compression test (c) bending test (d) torsion test respectively) (An et al.,1999).

Compression strength is the ability of the bone to withstand loads which tends to reduce its size i.e. resistance to pushing, while tensile strength is the resistance to pulling. The compression test technique straight away the mechanical indices and also calculates the mechanical properties, such as the elastic modulus, energy absorption, and structural rigidity by adopting simple and stressful conditions. In normal physiology the bone is supports the living body, hence the tensile forces are very small so, the tensile test is usually a complement to the compression test. Depending on bending tests the skeleton mechanical properties of the bone are measured in small animals. Using torsion tests of the biomechanics of tubular bones of animals has been estimated (Kong et al.,2020) This method requires actual bone sample of human to be tested. Taking the bone out of the body of a human being to test its mechanical properties is not feasible, hence determining bone mechanics using noninvasive techniques is of great importance.

\subsection{Non - Invasive Techniques For Bone Mechanics:} Quality of bone together with structural mechanical indices which depends on geometric shape of the bone and material mechanical which is dependent on components of the bone is usually represented by the mechanical characteristics of bone. These indices specify the bone strength and its capability to withstand external forces. The biomechanics of bones can be estimated using FEM along with the densitometric and structural information (Muszy囚ski et al.,2017) which can be obtained from imaging techniques such as DEXA, QCT etc.

2.2.1 Regression SVM: A classifier classifies some extracted features for example from a bone image and provides label to it. 20 different classifiers such as MLP, SMO, Random Tree etc are there for detection of osteoporosis which may use different feature selection criteria (Sapin-de et al.,2012) According to (Dubousset et al.,2005) there are around 27 variables such as BMI, height, weight, protein and calories consumption, calcium, exposure to sun light etc which affects BMD.

These variables were determined using regression trees applied to the support vector machines for predictions. Determining the BMD of bone, bone mass loss and to find out which variable has greatest effect on BMD is of great importance. Increasing calcium intake, maintaining BMI, daily exercise, more exposure to sunlight etc are the remedies to decrease the bone mass loss and in turn to improve the BMD of bone. To estimate BMD a questionnaire on diet and lifestyle and a mathematical model designed using regression SVM can be used. SVM have very good performance on classification and can also be used for continuous measurement of cortical width. But computationally SVMs does not work very efficiently, for large dataset.

2.2.2 Low Dose Imaging Technique: Low radiation dose imaging technique such as EOS Imaging, Paris E. (Sapin-de Brosses et al.,2012) (Dubousset et al.,2005) can be used for determining vertebral strength using subject specific FEM. 79\% of vertebral failure load is within the range of $\pm 734 \mathrm{~N}$, this has been convicted with 95\% using subject specific FEM. Also by implementing subject specific Young's modulus the relative error decreases by a good amount (El et al.,2012) This method requires improvements in material properties such as heterogeneity and anisotropy to estimate the result associated with failure criteria.

\subsubsection{Quantitative Computed Tomography (OCT):} Quantitative computed tomography (QCT) is an imaging technique which measures BMD using a standard X-ray Computed Tomography (CT) scanner. The Hounsfield Units (HU) of the CT images are converted to bone mineral density values with a calibration standard. QCT is basically used to estimate volumetric BMD at the lumbar spine and hip. Nowadays, modern 3D QCT are being used to obtain three-dimensional images of the human body. Phantom less QCT (pQCT) and Multidetector QCT (MDCT) are mainly used for determination of bone mechanics. The BMD obtained by pQCT and MDCT is comparable with the BMD obtained by DEXA imaging technique (Pickhardt et al.,2011) QCT lumbar spine and DEXA lumbar spine have good correlation.

Also the feasibility of BMD measurements obtained from QCT has been studied (Mao et al.,2016) QCT has good accuracy in determining BMD, high precision and less accuracy error. Lumbar spine, hear phantom less scan, phantom heart scans etc can be used for estimating BMD, microarchitecture and in turn mechanical property of bone. The main cause of hip fracture is osteoporosis which makes the bones porous and more fragile. To find fracture risk and to provide prevention it is necessary to find strength of bone. A simulation model using CT based FEM will have good accuracy in predicting bone strength as compared to other models. 3D structural analysis can also be done using the simulation model based on CT based FEM.

For non-linear Finite Element Analysis (Beesho et al.2007) developed a software "Mechanical Finder" whose results were verified using Mechanical loading test using Universal Testing Machine (Bessho et al.,2007) QCT is more sensitive in finding bone density changes as compared to DEXA, but has high radiation; hence QCT is an alternative to DEXA (Burghardt et al.,2011) 
DEXA is a low cost, low radiation imaging technique and hence preferred over other imaging technique for the measurement of BMD.

2.2.4 Dual-Energy X-Ray Absorptiometry (DEXA): Dualenergy X-ray absorptiometry scan is a standard way for diagnosis of osteoporosis, it determines the bone density to evaluate the strength of the bone. DEXA works by directing two low- dose X-rays which gets absorbed in different ways by soft tissues and bones. If the bone density is less then there is more risk of fracture. The radiation of DEXA is very less, about $10 \%$ of a normal chest X- ray. DEXA is mostly used for determination of BMD at hip and lumbar spine region [Anon]. QCT gives volumetric BMD whereas DEXA gives areal BMD. DEXA has an advantage of low radiation dose and low cost but it has a disadvantage also that it cannot give details about microarchitecture and mechanical properties of bone (Dall'Ara et al.,2016).

Hence there is a need for such DEXA based technique which can easily predict bone fracture load. To fulfil this need subject specific FEM can be used (Dall'Ara et al.,2012) Simulated DEXA based FEM model was developed which was capable of predicting fracture load (Lu et al.,2019) This model has a limitation that it has used simulated DXA images ie. 2D coarsened projection of HR-pQCT images. Future scope is to develop a methodology and validate it for clinical DEXA scans. A new parameter called as Strain Index of Bone (SIB) was developed using FEM and was correlated with bone strength (Colombo et al.,2019) This parameter can also estimate bone fragility index. The vertebra strength predicted by SIB is better as compared to BMD, but this method also has a drawback that it could work better if 2D model was used and it is difficult to apply on human vertebrae as shape of human vertebrae is complex.

2.2.5 Finite Element Modelling: Even though the study of mechanical properties of bones using mechanical test approach is reliable and has been used widely its practical implementation has many inadequacies. Nowadays it is possible to theoretically simulate biomechanics of bone using the FEM. Due to the development in computer technology and modeling Etsimulating software, the FEM is finding large application in the area of biomechanics (Hao et al., 2011) widely used to analyze the mechanical response of biomaterials (Xiao et al.,2016) FEM for bones are categorized into two categories, one is $2 \mathrm{D}$ bone model and other one is 3D bone model. Basic block diagram of FEM to analyse bone mechanics with 3 basic steps: input, processing data and output data is shown in (Figure 3) (Claes et al.,2012).

2D Modelling: The 2D bone model is comparatively simple to setup. The proximal and distal bones of the femur were mesh by a 3-node point-membrane unit and the author's own algorithm was used for studying the distribution of 2D bone density (Chen et al.) The results obtained were compared with the experimental results and found to be reliable. If not, then another capable tool to get more accurate assessment of bone is 2D FEM based on DXA image (Luo et al.,2013). As compared to 3D models there is a chance of loss of data in using 2D models (Quevedo et al.,2017) Hence, a 3D FE skeletal model has been developed which is very close to a real skeletal model.

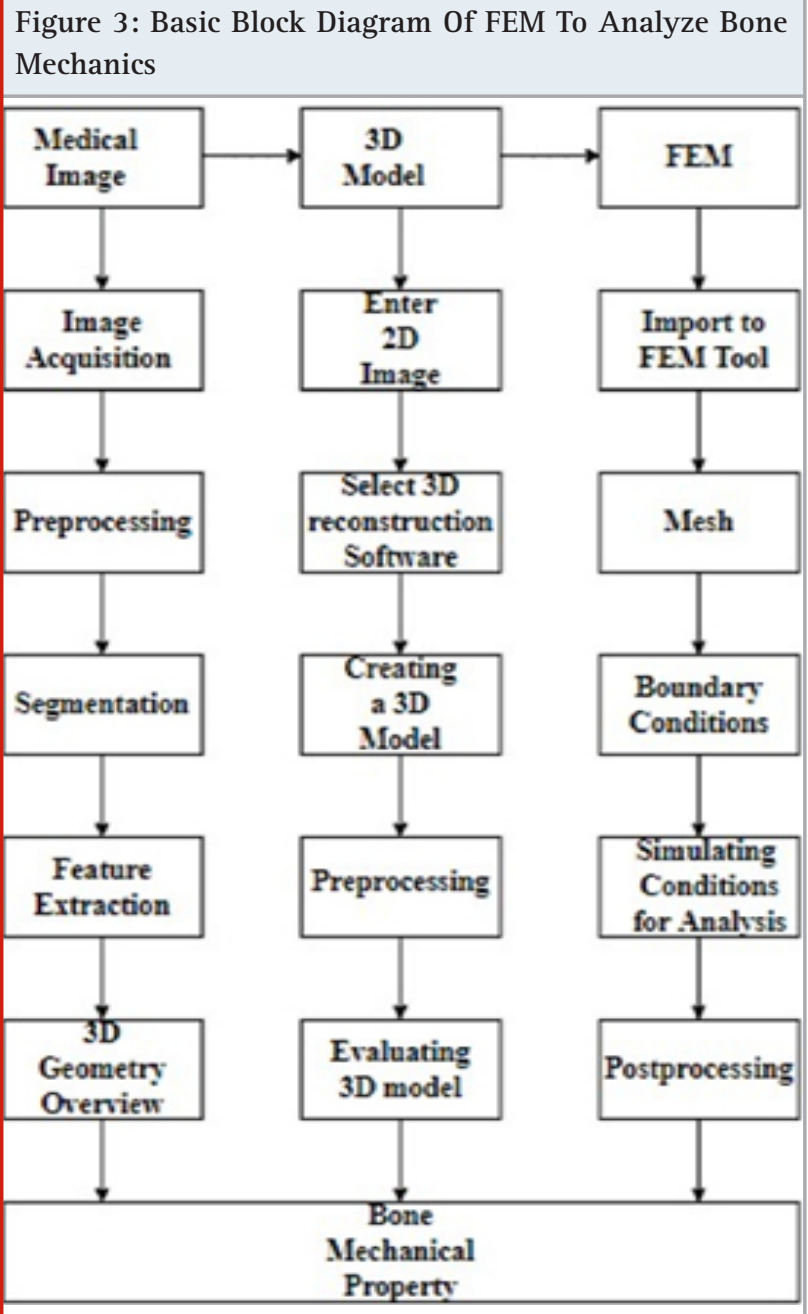

3D Modelling: 3D finite element modelling is mainly used to analyse stress, strain and bone fracture risk (Väänänen et al.,2015) There are different modelling methods according to which the 3D FEM can be divided into following types:

Geometric Modelling: A 3D parametric CAD model using SolidWorks ${ }^{\circledR}$ 2017, USA has been developed by (Cali et al.,2018) Further a 3D FEM for objects with regular shape has been constructed as per the geometry and size of an object. But, the structure of human body is complex and has irregularity in geometric shapes which makes it difficult to create models using this method. 
3D Scanner Modelling: (Gok et al.,2017) used 3D scanner and used 3D laser scanning technology to obtain a point cloud after scanning the human femoral model, which was converted to a 3D femoral model using the software Geomagic Studio. 3D Scanner Modelling gave more accurate results, but irregularity in geometric shapes, makes it difficult to get the point cloud file of the internal structure.

3D Modelling Based On Dexa Image: DEXA image based FEM is a capable tool to get more accurate assessment of bone (Luo Y et al.,201 ( S P et al.,2015) designed one technique using a 2D BMD image and a femur shape template to define the 3D shape and density distribution of the proximal femur. (Thevenot et al.,2014) designed one technique to mechanically construct a patient- specific 3D FEM using standard 2D radiographs, unparalleled prospective for determination of patientspecific failure loads.

CT Modelling: Due to the large increase in use of CT, 3D CT scan images are easily available nowadays. Software such as Mimics can be used for medical reconstruction to form the 3D solid models. This method is appropriate to model irregular objects, and is the conventional method for developing 3D FEM (Soodmand et al.,2018), (Ni J et al.,2018) Certain researchers say that as compared to DEXA the CT modelling generate excess radiation on patients, which limits its usefulness in practical implementations. But the DEXA model also needs to be improved especially in terms of accuracy.

2.2.6 Buckling Ratio: Buckling ratio captures compensation mechanism and is the ratio of outer radius to cortical thickness. Critical fracture load FCR can be derived using FEA which captures compressive strength of femur (Anitha et al.,2014) More accurate analysis of QCT scans for improving the diagnosis can be provided by incorporating FCR and Buckling Ratio with BMD. FCR relates with BMD and Buckling Ratio as, higher the higher is the BMD and lower is the Buckling Ratio.

2.2.7 Density - Elasticity Relationship: Impact of loading conditions on bone which in turn creates different strain and stress leading towards fracture are difficult to predict. Subject specific finite element analysis can be used to determine whether accurate strain can be predicted or not using Density - elasticity relation. Density - elasticity has greater impact on accuracy of strain. There are 3 different density - elasticity relationships which can predict stain/stress (Schileo et al.,2007)

\section{RESULTS}

The different parameters were studied for measurement of mechanical load distribution and its impact on bone strength. These biomechanical tests attribute towards the major factors of bone strength in terms of tensile strength, mechanical shear, presence of multiple diseases can also be considered while analyzing bone strength. Also it is clear from the study that the FE analysis is highly useful for bone strength estimation.

\section{DISCUSSION}

One of the most important limitations of FE model is lack of anatomical details, which can be overcome with the improvement in computer tomography. But computer tomography is time consuming. This difficulty can be overcome to some extent using X-ray/DEXA images. With the estimation of bone mechanics using FEM, one can determine the strength or fracture point of patient. Hence can prevent fracture to some extent or provide the required treatment in order to improve the strength of bone. In future one may perform strength calculation or fracture risk prediction non-invasively and use $2 \mathrm{D}$ and 3D models for validation as it produces highly precise results. Instead of using simulated DEXA or QCT we are using DEXA as it is low cost low radiation and easily available technique.

\section{CONCLUSION}

This study is of clinical importance and it clearly highlights the diagnostic tests for analysis of mechanical loads that determine bone strength. The increase in various factors of stress and strain can result in escalated mechanical load and thus result in fractures and osteoporosis. The present study had some limitations; firstly it did not specify a particular population upon which these tests can give best results in terms of gender, age and presence of multiple diseases. Second, the efficacy of results is not in statistical data and thus strength factors may vary due to individual mechanical load distribution for avoiding fractures. Also the time of duration for which this intervention must be used to check the mechanical load that impact bone strength was not specified. But the tests for mechanical load testing and analysis of the impact on bone strength occur mainly due to aging progression as well as multiple diseases.

The major changes in mechanical properties due to high stress strain relation proportionality occur in cortical bone, trabecular bone, as well as whole bones. Another major factor that impacts bone strength due to increased mechanical load is due to porosity which is of high clinical significance. The multitude of bonequality parameters is another contributing factor which leads to poor balance and increased mechanical load on specific point. The research findings are aligned with biological hormonal interactions which impact bone strength and movement. These mechanical tests have nano scale impact and significance but can result in huge interventionary models for corrective therapies of 
bone deformities and cracks due to fatigue and stress and strain escalation. Thus diagnostic methods for mechanical load help in mitigating risks of fractures due to aging, hormonal changes and multiple diseases.

Conflict of Interest: There is no conflict of interest among authors.

\section{ACKNOWLEDGEMENTS}

Authors sincerely acknowledge accendere research coordinators for their support.

Financial Disclosure: This work is not funded by any funding agency.

\section{REFERENCES}

An, Y.H., Draughn, R.A. and Draughn, R.A. (1999). Mechanical Testing of Bone and the Bone-Implant Interface. CRC Press. Available from: https://www. taylorfrancis.com/books/e/9780429125799.

Anderson, A.E., Peters, C.L., Tuttle, B.D. and Weiss, J.A. (2005). Subject-Specific Finite Elepment Model of the Pelvis: Development, Validation and Sensitivity Studies. Journal of Biomechanical Engineering, 127(3): 364-373.

Anitha, D. and Lee, T. (2014). Assessing Bone Quality in Terms of Bone Mineral Density, Buckling Ratio and Critical Fracture Load. Journal of Bone Metabolism, 21(4): 243.

Bessho, M., Ohnishi, I., Matsuyama, J., Matsumoto, T., Imai, K. and Nakamura, K. (2007). Prediction of strength and strain of the proximal femur by a CT-based finite element method. Journal of Biomechanics, 40(8): 1745-1753.

Burghardt, A.J., Link, T.M. and Majumdar, S. (2011a). High-resolution Computed Tomography for Clinical Imaging of Bone Microarchitecture. Clinical Orthopaedics and Related Research ${ }^{\circledR}$, 469(8): 2179-2193.

Cali, M., Zanetti, E.M., Oliveri, S.M., Asero, R., Ciaramella, S., Martorelli, M. and Bignardi, C. (2018). Influence of thread shape and inclination on the biomechanical behaviour of plateau implant systems. Dental Materials, 34(3): 460-469.

Chen, B., Zhao, W. and Sun, Y. (2008). Bone remodeling numerical simulation on the basis of bone adaptive theory. Journal of Biomedical Engineering, 25(2): 363-367.

Claes, L., Recknagel, S. and Ignatius, A. (2012). Fracture healing under healthy and inflammatory conditions. Nature Reviews Rheumatology, 8(3): 133-143.

Colombo, C., Libonati, F., Rinaudo, L., Bellazzi, M., Ulivieri, F.M. and Vergani, L. (2019). A new finite element based parameter to predict bone fracture. Fyhrie, D., ed. PLOS ONE, 14(12): e0225905.

Dall'Ara, E., Eastell, R., Viceconti, M., Pahr, D. and Yang, L. (2016). Experimental validation of DXA-based finite element models for prediction of femoral strength. Journal of the Mechanical Behavior of Biomedical Materials, 63: 17-25.

Dall'Ara, E., Pahr, D., Varga, P., Kainberger, F. and Zysset, P. (2012). QCT-based finite element models predict human vertebral strength in vitro significantly better than simulated DEXA. Osteoporosis International, 23(2): 563-572.

Dual energy X-ray absorptiometry - Bone mineral densitometry. (2017). Available from: https://www. iaea.org/resources/rpop/health-professionals/otherspecialities-and-imaging-modalities/dxa-bonemineral-densitometry.

Dubousset, J., Charpak, G., Dorion, I., Skalli, W., Lavaste, F., Deguise, J., Kalifa, G. and Ferey, S. (2005). A new 2D and 3D imaging approach to musculoskeletal physiology and pathology with low-dose radiation and the standing position: the EOS system. Bulletin De l'Academie Nationale De Medecine, 189(2): 287-297.

El Masri, F., Sapin de Brosses, E., Rhissassi, K., Skalli, W. and Mitton, D. (2012). Apparent Young's modulus of vertebral cortico-cancellous bone specimens. Computer Methods in Biomechanics and Biomedical Engineering, 15(1): 23-28.

Gok, K., Inal, S., Gok, A. and Gulbandilar, E. (2017). Comparison of effects of different screw materials in the triangle fixation of femoral neck fractures. Journal of Materials Science: Materials in Medicine, 28(5): 81. Grassi, L., Väänänen, S.P., Ristinmaa, M., Jurvelin, J.S. and Isaksson, H. (2017). Prediction of femoral strength using 3D finite element models reconstructed from DXA images: validation against experiments. Biomechanics and Modeling in Mechanobiology, 16(3): 989-1000.

Hao, Z., Wan, C., Gao, X. and Ji, T. (2011). The Effect of Boundary Condition on the Biomechanics of a Human Pelvic Joint Under an Axial Compressive Load: A Three-Dimensional Finite Element Model. Journal of Biomechanical Engineering, 133(10): 101006.

Kong, D., Shi, Y., Lin, G., Jiang, B. and Du, J. (2020). Recent Advance in Evaluation Methods for Characterizing Mechanical Properties of Bone. Archives of Computational Methods in Engineering, 27(3): 711-723.

Lopes, V.M.M., Neto, M.A., Amaro, A.M., Roseiro, L.M. and Paulino, M.F. (2017). FE and experimental study on how the cortex material properties of synthetic femurs affect strain levels. Medical Engineering \& Physics, 46: 96-109.

Lu, Y., Zhu, Y., Krause, M., Huber, G. and Li, J. (2019). Evaluation of the capability of the simulated dual energy X-ray absorptiometry-based two-dimensional finite element models for predicting vertebral failure loads. Medical Engineering \& Physics, 69: 43-49.

Luo, Y., Ferdous, Z. and Leslie, W.D. (2013). Precision study of DXA-based patient-specific finite element modeling for assessing hip fracture risk: PRECISION OF DXA-BASED FINITE ELEMENT MODELING. 
International Journal for Numerical Methods in Biomedical Engineering, 29(5): 615-629.

Mao, S.S., Li, D., Luo, Y., Syed, Y.S. and Budoff, M.J. (2016). Application of quantitative computed tomography for assessment of trabecular bone mineral density, microarchitecture and mechanical property. Clinical Imaging, 40(2): 330-338.

Muszynski, S., Kwiecien, M., Tomaszewska, E., \wietlicka, I., Dobrowolski, P., Kasperek, K. and Jezewska-Witkowska, G. (2017). Effect of caponization on performance and quality characteristics of long bones in Polbar chickens. Poultry Science, 96(2): 491-500.

Ni, J., Li, D., Mao, M., Dang, X., Wang, K., He, J. and Shi, Z. (2018). A Method of Accurate Bone Tunnel Placement for Anterior Cruciate Ligament Reconstruction Based on 3-Dimensional Printing Technology: A Cadaveric Study. Arthroscopy: The Journal of Arthroscopic \& Related Surgery, 34(2): 546-556.

Op Den Buijs, J. and Dragomir-Daescu, D. (2011). Validated finite element models of the proximal femur using two-dimensional projected geometry and bone density. Computer Methods and Programs in Biomedicine, 104(2): 168-174.

Parashar, S.K. and Sharma, J.K. (2016). A review on application of finite element modelling in bone biomechanics. Perspectives in Science, 8: 696-698.

Pickhardt, P.J., Lee, L.J., Muñoz del Rio, A., Lauder, T., Bruce, R.J., Summers, R.M., Pooler, B.D. and Binkley, N. (2011). Simultaneous screening for osteoporosis at CT colonography: Bone mineral density assessment using MDCT attenuation techniques compared with the DXA reference standard. Journal of Bone and Mineral Research, 26(9): 2194-2203.

Quevedo González, F.J., Reimeringer, M. and Nuño, N. (2017). On the Two-Dimensional Simplification of Three-Dimensional Cementless Hip Stem Numerical Models. Journal of Biomechanical Engineering, 139(3): 031011.

Sapin-de Brosses, E., Jolivet, E., Travert, C., Mitton,
D. and Skalli, W. (2012). Prediction of the Vertebral Strength Using a Finite Element Model Derived From Low-Dose Biplanar Imaging: Benefits of SubjectSpecific Material Properties. Spine, 37(3): E156-E162. Schileo, E., Taddei, F., Malandrino, A., Cristofolini, L. and Viceconti, M. (2007). Subject-specific finite element models can accurately predict strain levels in long bones. Journal of Biomechanics, 40(13): 2982-2989.

Soodmand, E., Kluess, D., Varady, P.A., Cichon, R., Schwarze, M., Gehweiler, D., Niemeyer, F., Pahr, D. and Woiczinski, M. (2018). Interlaboratory comparison of femur surface reconstruction from CT data compared to reference optical 3D scan. BioMedical Engineering OnLine, 17(1): 29.

Taghizadeh, E., Kistler, M., Büchler, P. and Reyes, M. (2016). Fast Prediction of Femoral Biomechanics Using Supervised Machine Learning and Statistical Shape Modeling. In: Joldes, G. R. et al., eds. Computational Biomechanics for Medicine. Cham: Springer International Publishing: 107-116. Available from: http://link. springer.com/10.1007/978-3-319-28329-6_10.

Thevenot, J., Koivumäki, J., Kuhn, V., Eckstein, F. and Jämsä, T. (2014). A novel methodology for generating 3D finite element models of the hip from 2D radiographs. Journal of Biomechanics, 47(2): 438-444.

Väänänen, S.P., Grassi, L., Flivik, G., Jurvelin, J.S. and Isaksson, H. (2015). Generation of 3D shape, density, cortical thickness and finite element mesh of proximal femur from a DXA image. Medical Image Analysis, 24(1): 125-134.

Xiao, W., Zaeem, M.A., Bal, B.S. and Rahaman, M.N. (2016). Creation of bioactive glass (13-93) scaffolds for structural bone repair using a combined finite element modeling and rapid prototyping approach. Materials Science and Engineering: C, 68: 651-662.

Yu, E.W., Bouxsein, M.L., Roy, A.E., Baldwin, C., Cange, A., Neer, R.M., Kaplan, L.M. and Finkelstein, J.S. (2014). Bone Loss After Bariatric Surgery: Discordant Results Between DXA and QCT Bone Density: QCT AND DXA BONE DENSITY AFTER BARIATRIC SURGERY. Journal of Bone and Mineral Research, 29(3): 542-550. 